\title{
Forschung kurz notiert
}

\section{Neugeborenenscreening - Verwendung von Rest- material für Forschungs- zwecke?}

In einer US-amerikanischen Studie wurde die öffentliche Meinung zu der Frage, ob verbleibendes Restmaterial (Blut) aus dem Stoffwechselscreening für Forschungszwecke verwendet werden soll, untersucht. Zu diesem Zweck wurden 3855 Personen mit einem strukturierten Fragebogen befragt. Knapp die Hälfte der Befragten bekam einen 22-minütigen Film zur Ansicht, in welchem erklärt wurde, wofür die Blutproben verwendet werden. Die Mehrheit aller Befragten äußerte Zustimmung zur Verwendung des Blutrestmaterials für Forschungszwecke, wobei das vorherige Ansehen des Informationsfilms den Prozentsatz noch erhöhte. Allerdings sprach sich die Mehrheit für ein „opt-in“ aus, also für eine Verwendung nur nach ausdrücklicher Erlaubnis durch die Eltern.

Pediatrics 2012; 129:231-238 PMID 22250018

\section{Immunthrombozytopenie} nach Impfung

In einer amerikanischen retrospektiven Kohortenstudie, welche 1,8 Mio. Kinder und Jugendliche einschloss, wurde das Risiko für das Auftreten einer Immunthrombozytopenie (ITP) nach Impfung untersucht. Die Diagnose ITP, deren Schweregrad und Verlauf wurden anhand der Krankengeschichten objektiviert. Insgesamt wurden 197 ITP-

Monatsschr Kinderheilkd 2012 - 160:620-620 DOI 10.1007/s00112-012-2672-y

\section{Redaktion:}

O. Bodamer (Miami), J. Freihorst (Aalen),

R. Kerbl (Leoben), G. Krandick (Oberhaching)
Fälle identifiziert. Ein erhöhtes Risiko für das Auftreten einer ITP innerhalb von 6 Wochen nach der Impfung ergab sich für die Masern-Mumps-Röteln-Impfung (MMR). Statistisch erhöht (jedoch nur auf einigen wenigen Fällen beruhend) war auch das Risiko nach Hepatitis-A-Impfung bei 7- bis 17-Jährigen sowie für Varizellen- und DiphtherieTetanus-Pertussis-Impfung bei 11- bis 17-Jährigen. Die Verläufe waren vorwiegend akut, mild und ohne Langzeitfolgen.

Pediatrics 2012; 129:248-255

PMID 22232308

\section{Antibiotische Therapie der ersten Pyelonephritis im Kindesalter}

In einer französischen prospektiven Multicenterstudie wurden die orale und die i.v. Behandlung der ersten Pyelonephritis von Säuglingen und Kleinkindern (Alter: 1 bis 36 Monate) im Hinblick auf bleibende Nierenparenchymschädigung nach szintigraphisch gesicherter akuter Nierenbeteiligung untersucht. Die Behandlung erfolgte randomisiert mit oralem bzw. i.v. Zephalosporin. Die Parenchymnarben wurden 6 bis 8 Monate später szintigraphisch objektiviert. Dabei ergab sich zwischen den beiden Gruppen kein signifikanter Unterschied (27,3\% in der i.v. behandelten Gruppe, 30,8\% in der oral behandelten Gruppe). Die Autoren folgerten, dass die primäre orale antibiotische Behandlung bei erster Pyelonephritis ohne präexistente Harnwegfehlbildung weitgehend der i.v. Behandlung gleichwertig ist.

Pediatrics 2012; 129:e269-e275

PMID 22291112

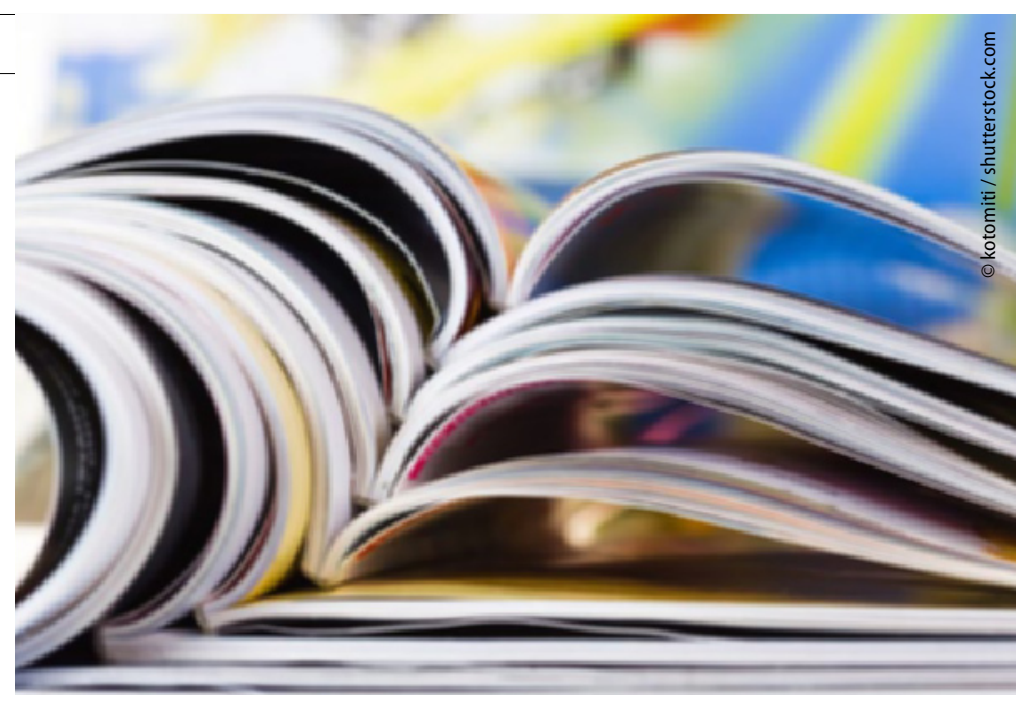

Montelukast - wirkungslos in der Prävention von Infekten der oberen Luftwege Virale Infektionen der oberen Luftwege gehen mit erhöhten Leukotrienspiegeln einher. In einer israelischen Studie wurde daher untersucht, ob der prophylaktisch verabreichte Leukotrienrezeptorantagonist Montelukast in der Lage ist, derartige Episoden zu verhindern. In einer randomisierten plazebokontrollierten Doppelblindstudie erhielten 153 Kinder (Alter 1 bis 5 Jahre) über 12 Wochen täglich $4 \mathrm{mg}$ Montelukast und 147 Kinder ein gleich aussehendes Plazebo. Die Eltern der Kinder wurden wöchentlich telefonisch kontaktiert und über das Auftreten respiratorischer Infektionen befragt. In der mit Montelukast behandelten Gruppe wurde in 30,4\% der Beobachtungswochen eine Atemwegsinfektion registriert, in der Plazebogruppe in $30,7 \%$ der Beobachtungswochen. Somit ergab sich für Montelukast keine signifikante präventive Wirkung.

Pediatrics 2012; 129:e285-e290

PMID 22218843

\section{Ethanol-Lock für zentrale} Venenkatheter als Schutz vor Kathetersepsis

In einer Metaanalyse einer kanadischen Autorengruppe wurde das Auftreten katheterbedingter
Infektionen bei Verwendung eines Ethanol-Lock im Vergleich mit dem herkömmlichen Heparin-Lock verglichen. Für den Zeitraum von 1948-2010 wurden 4 vergleichende Studien zu dieser Fragestellung gefunden. Dabei zeigte sich, dass bei Verwendung des Ethanol-Locks weniger katheterbedingte Infektionen auftraten. Es wurde errechnet, dass auf 1000 Kathetertage 7,7 Infektionen und 5,07 durch Infektion erzwungene Katheterwechsel vermieden werden konnten, dies entspricht einer Reduktion um $81 \%$ für Infektionen bzw. um 72\% für Katheterwechsel. Als unerwünschte Nebenwirkung wurden allerdings vereinzelt thrombotische Ereignisse beobachtet.

Pediatrics 2012; 129:318-329

PMID 22232307

\section{Korrespondenzadresse}

Prof. Dr. R. Kerbl

Abteilung für Kinder und Jugendliche LKH Leoben

Vordernbergerstraße 42 8700 Leoben, Österreich reinhold.kerbl@lkh-leoben.at 